\title{
THE GROWTH OF A PASSIVE FILM ON STEEL STUDIED WITH IN-SITU AFM
}

\author{
GERRIT ZIJLSTRA, ENNE T. FABER, VÁCLAV OCELÍK \& JEFF TH. M. DE HOSSON \\ Department of Applied Physics, University of Groningen, The Netherlands
}

\begin{abstract}
The passive film on stainless steel is a crucial barrier to retard or block further corrosion of the bulk. After damage due to fabrication processes or during use in service, the passive film has to be recovered. Little is known of the characteristic time scale of the passivation process in ambient conditions, i.e. air. In this work, a quantitative method is presented to follow the growth of the passive layer after damage, in-situ by AFM. The passive layer of a defined area is removed by sputtering with argon ions. The recovery of the film is measured by recording the height difference between the sputtered area and the reference area using AFM. The growth of the new passive film is terminated after 2 hours. The height difference of the passive film and the substrate is measured 25 minutes after exposure to air and extrapolated to be 21 nanometers at the onset of passivation. This thickness agrees rather well with the calculated range of 18.9 to 21.5 nanometers.
\end{abstract}

Keywords: steel, recovery, AFM, oxidation, iron, chromium, passive layer, thin film.

\section{INTRODUCTION}

Most metals, but more specific stainless steel, exhibit a passive layer, which protects the bulk from further corrosion. In general, the structure and composition of this layer depends on the conditions present at formation, e.g. dry-, wet-, chloride containing environment or acid solutions, for which the thickness can vary from 10 to $40 \AA$ [1]. A well-known technique to characterize this layer is to use X-ray photoelectron spectroscopy (XPS). The chemical composition of the passive layer can be obtained by depth profiling, due to a procedure of alternating surface scanning with XPS and layer removal with argon ions. The general picture for iron-chromium steel is a mixed film of iron- and chromium oxides: (Fe, $\mathrm{Cr})_{2} \mathrm{O}_{3}$ and $(\mathrm{Fe}, \mathrm{Cr})_{3} \mathrm{O}_{4}$ [2], with a thickness in the order of several nanometers [1], [3], [4]. Metals share the remarkable feature that the passive film has the ability to be self-healing. When for example the surface of iron-chromium steel is scratched and a part of the layer is removed, chromium from the bulk diffuses to the damaged area and a new passive layer is formed. Stainless steels therefore have an in-built self-repairing corrosion protection system.

Martensitic stainless steel is widely used for consumer products, e.g. in cutlery. Commonly used sequential processing steps with martensitic chromium steel are to apply metal forming achieving the desired shape and then apply a heat treatment to attain the required mechanical strength. Throughout this process and later during service, the film has to be re-established. However, this re-passivation takes time. As mentioned above, the thickness and composition depend on the environment during forming [1], which determines the quality and effective corrosion resistance of the passive film. It is key to keep the conditions optimal during passivation, but therefore one should know how long these conditions should be maintained.

In this paper, an experimental procedure with AFM is presented and discussed to determine the required passivation time in air. 


\section{EXPERIMENTAL}

The material is a martensitic stainless steel, with chemical composition listed in Table 1. Samples were given a mirror polish finish, where the final step included polishing with $1 / 4$ micron diamond particles. An even smoother result could have been obtained by finishing with e.g. colloidal silica particle suspension as OP-S or OP-U which are of a typical size of $40 \mathrm{~nm}$. However, these suspensions are water based, as opposed to the alcohol-type lubrication used for polishing with diamond particles. Recovery of the passive layer immediately after polishing would thus partly take place under very wet conditions, not resembling recovery in regular atmospheric conditions, which could affect the typical composition and thickness of the passive layer. After polishing with $1 / 4$ micron diamond particles, the surface was rinsed with ethanol and dried with warm air. Thereafter samples were placed for 7 days in a controlled environment with $50 \%$ relative humidity and a temperature of $21^{\circ} \mathrm{C}$, in order to restore a stable passive layer.

The passive layer can be removed by sputtering (bombarding) the oxides with argon ions. Therefore, a sample was placed on a XPS sample-holder, as shown in Fig. 1. The upper part of the sample was shielded by a tantalum foil. The foil allows for close placement at the surface (without touching), to exclude shadowing and side effects during sputtering/bombardment with argon ions. The sputtering gun has an incident angle with the surface of 45 degrees, releasing $\mathrm{Ar}^{+}$ions with energy of $2 \mathrm{keV}$. No large differences in oxide removal is expected, as the sputter rate of $\mathrm{Fe}_{2} \mathrm{O}_{3}$ and $\mathrm{Cr}_{2} \mathrm{O}_{3}$ are 0.62 and 0.54 respectively, relative to sputtering of $\mathrm{SiO}_{2}$ [5]. The argon beam is rasterizing over the whole sample holder area, which provides an equal exposure to $\mathrm{Ar}^{+}$ions. Sputtering took place in an UHV preparation chamber, which was coupled to an XPS chamber. During the ion bombardment, the pressure in the chamber increased from $10^{-9}$ to $10^{-7} \mathrm{mbar}$, caused by the leakage of argon ions. Nevertheless, the partial pressure of oxygen was too low for short term re-passivation of the bare substrate.

Sputtering was periodically interrupted to perform an XPS scan to probe the surface composition. In this way, it is verified that the passive film is removed, and unnecessary sputtering of the metal-bulk-substrate is prevented. For the specimen considered in this work and three test samples, this point was reached after 15 minutes sputtering. The border between the sputtered and not-sputtered area is clearly visible by eye and under the optical microscope, as illustrated in Fig. 2.

Atomic force microscopy (AFM) was conducted with a Veeco Dimension 3100 with sub-nanometer resolution. The contrast between the two regions of Fig. 2 aids in finding the appropriate scanning position with the optical microscope of the AFM. An area of $25 \times 25$ micron was sampled in normal tapping mode, with 512 x 512-line resolution per image. Care was taken to place the sample such that the AFM scanning trace was perpendicular to the border, i.e. from left to right as in Fig. 2. The resulting trace can be seen in Fig. 3. Although the trace has not been leveled with a plane fit, it is clearly seen that, from left to right, the surface contains a step on the left of the center.

\begin{tabular}{|c|c|c|c|c|c|c|}
\hline $\mathrm{C}$ & $\mathrm{Cr}$ & $\mathrm{Si}$ & $\mathrm{Mn}$ & $\mathrm{P}$ & $\mathrm{S}$ & $\mathrm{Fe}$ \\
\hline 0.32 & 13.7 & 0.15 & 0.30 & $\leq 0.025$ & $\leq 0.010$ & Bal. \\
\hline
\end{tabular}

Table 1: Chemical composition of martensitic stainless steel (wt.\%). 


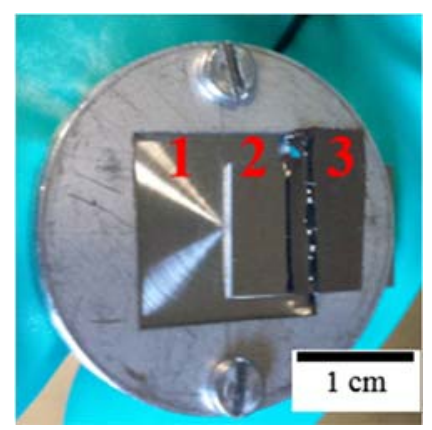

Figure 1: XPS sample holder (1), with sample (2) and tantalum foil shield (3).

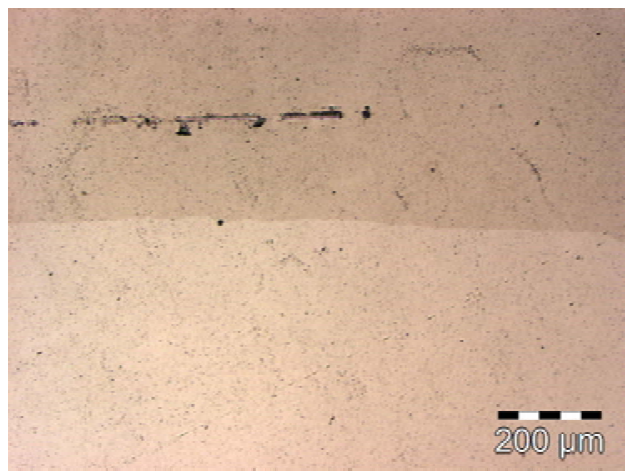

Figure 2: Optical image of the surface after sputtering. Light area (left) exposed to sputtering, dark area (right) shielded during sputtering.

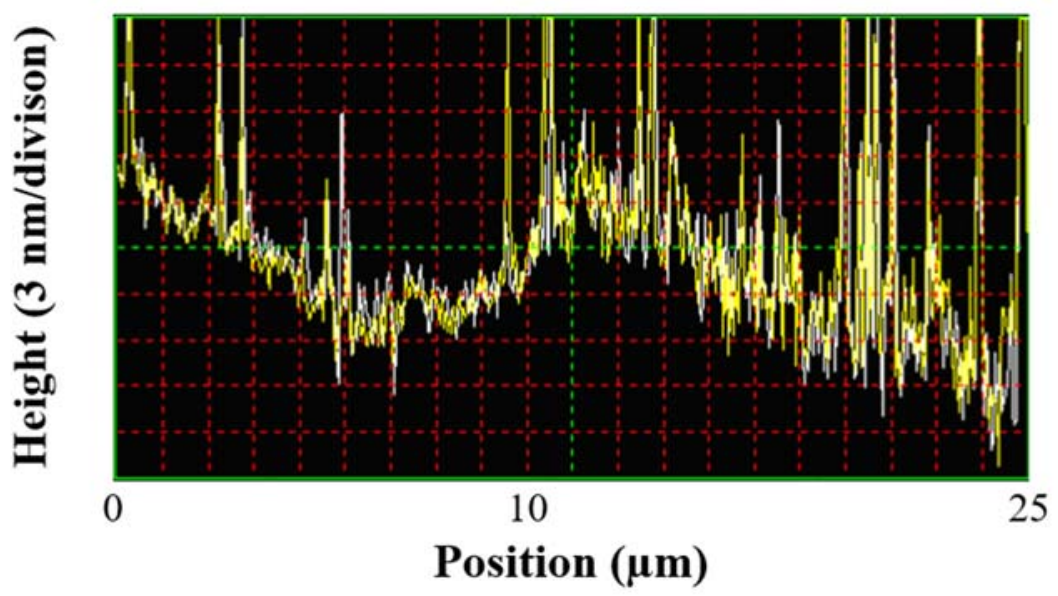

Figure 3: Typical trace with the step visible left of the center. 


\section{RESULTS AND DISCUSSION}

A complete $25 \times 25$ micrometer scan is shown in Fig. 4, with a plane fit for visualization purpose only. Visible over the whole substrate are scratches smaller than 25 nanometers, which are remnant from polishing. It is clear that the right-hand side has an elevation with respect to the left-hand side.

To quantify the step between these two areas, we defined a data processing method with computer code (Mathematica software) which uses the raw AFM $x, y$ and $z$ data as input. The $z$ values represent the height of the $x$ and $y$ plane, with $y$ defined as parallel to the border. For each $x$ position, the $z$ values of all corresponding $y$ points (i.e. a column) are averaged. In this way, the traces as in Fig. 3, are averaged for each of the $512 y$-entry's, in order to make a $2 \mathrm{D}$ projection of the topography. This projection is shown in Fig. 5 . The points in blue are used for a fit of the sputtered area, red for the not-sputtered. A third order polynomial function is fitted through the points of the step (in black). The $x$-value, where the first order derivative of this function changes the sign, is defined as the position of the step. The absolute difference between the two plane fits at this position is therefore taken as the height of the step.

The difference in color is also notable after several weeks of re-passivation. Therefore, the change in color (from dark to light, Fig. 4) is not only caused by the difference in passive layer thickness, but most likely also due to a difference in roughness.

In the following experiment the change of the step height after sputtering, due to recovery of the passive layer, will be presented. The sample was sputtered until the passive layer was removed. Thereafter the specimen was removed from the vacuum chamber and placed in the AFM, under ambient conditions of about $50 \% \mathrm{RH}$ and $21^{\circ} \mathrm{C}$. There the sputtering boundary had to be found and the AFM tip brought close to the surface. The first scan could be started 25 minutes after exposure, and took 20 minutes to complete. Immediately after finishing, a new scan was started on the same area. Following scans were made with an interval of one hour, as shown in Fig. 6. The step height decreases with time

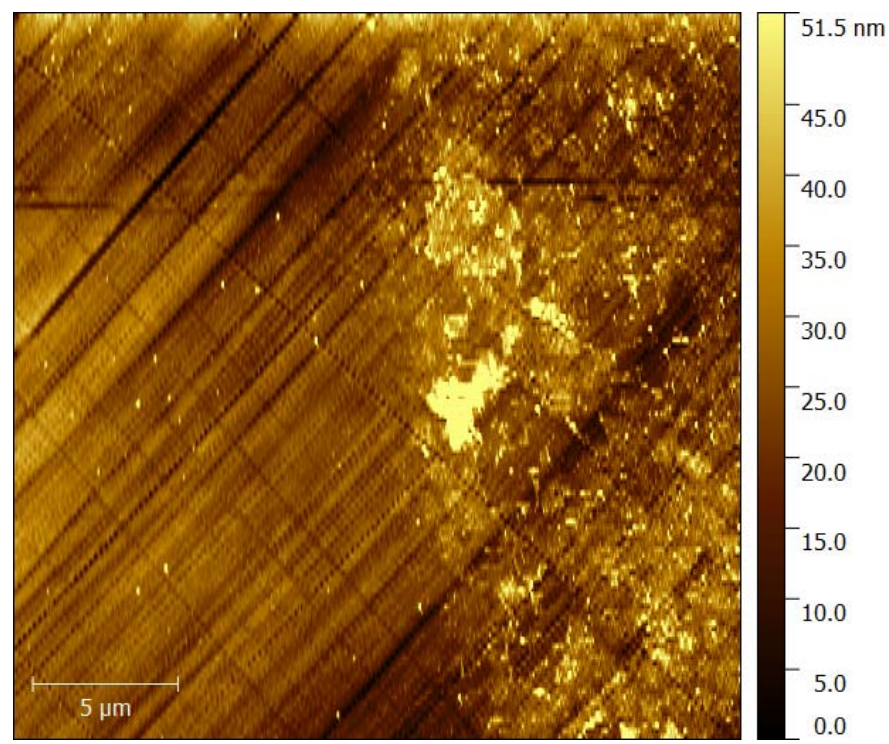

Figure 4: AFM topography map with sputtered section on the left side, and the elevated not-sputtered area on the right. 


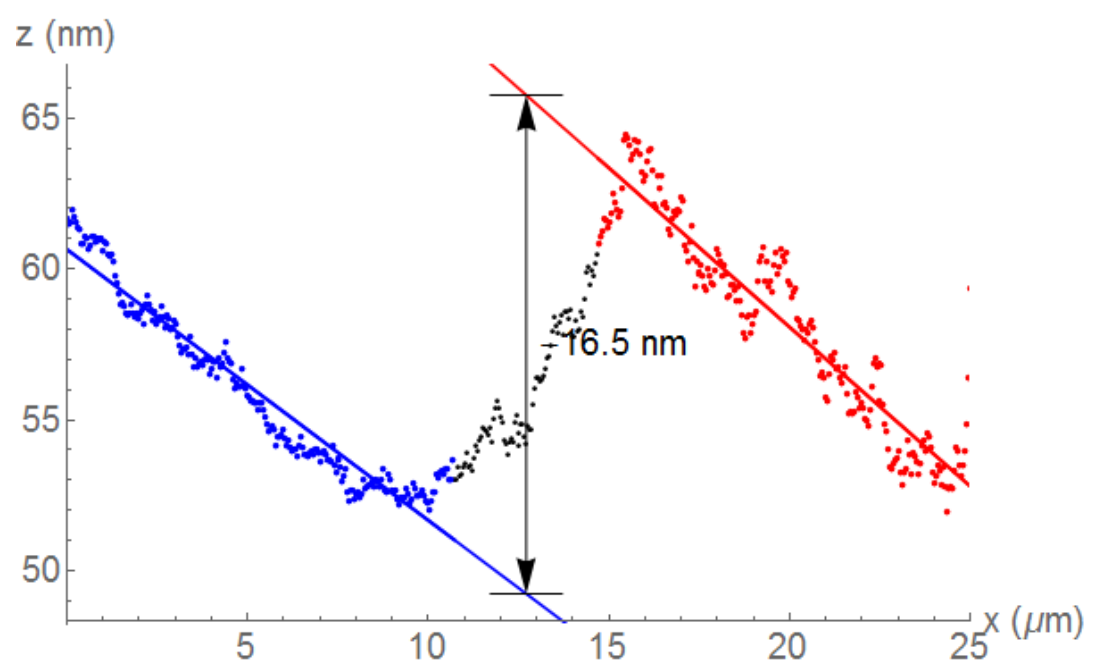

Figure 5: 2D projection of the topography, where blue represent a fit of the sputtered plane, red a fit of the not-sputtered plane.

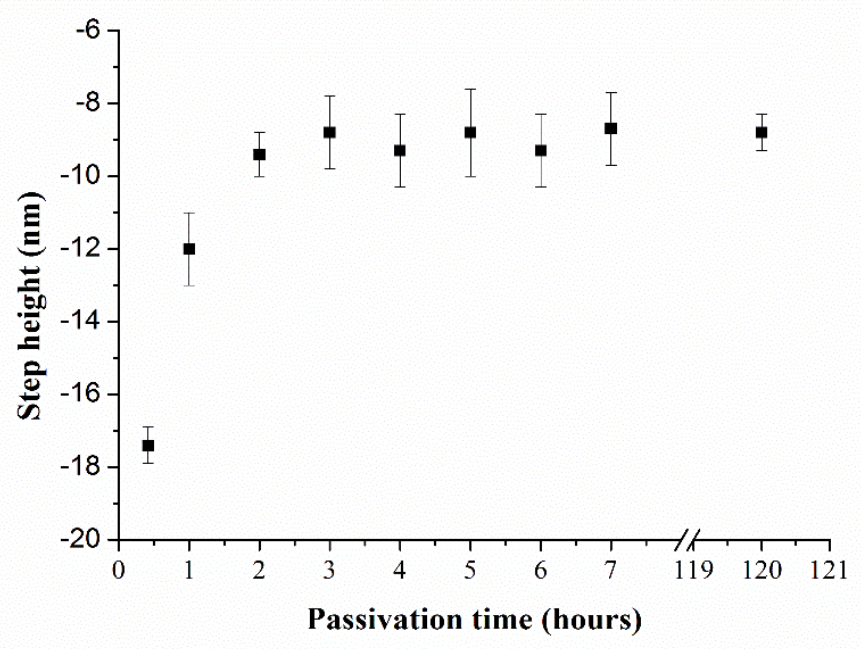

Figure 6: AFM recording of the decreasing of the step height, during recovery of the sputtered passive layer.

and stabilizes after 2 hours to a difference of -9 nanometers, confirmed by a measurement after 5 days.

Immediately after exposure to air, i.e. after removal from the vacuum chamber, the sputtered (bare) surface begins to oxidize. If we suppose that in the early stage of passivation $(<1$ hour) the growth of the layer is approximately linear, extrapolation of the 
first two points towards zero will give an initial step $(\mathrm{t}=0)$ of $-21 \mathrm{~nm}$. The passive layer consists of $\mathrm{Fe}_{3} \mathrm{O}_{4}, \mathrm{Fe}_{2} \mathrm{O}_{3}$, and $\mathrm{Cr}_{2} \mathrm{O}_{3}$, with density of 5180,5240 and $5210 \mathrm{~kg} / \mathrm{m}^{3}$ respectively [6], in contrast to $7874 \mathrm{~kg} / \mathrm{m}^{3}$ for pure iron. Therefore, a density ratio of $\rho_{o x} /$ $\rho_{\text {met }}=0.67$ exists for $\mathrm{Fe}_{2} \mathrm{O}_{3}(o x)$ and $\mathrm{Fe}$ (met), so the relation between the two becomes:

$$
\frac{m_{o x}}{V_{o x}}=0.67 \frac{m_{m e t}}{V_{m e t}}
$$

If we assume, for example, that the passive layer consists solely of $\mathrm{Fe}_{2} \mathrm{O}_{3}$, the ratio of the molecular masses is

$$
\frac{m_{o x}}{m_{m e t}}=\frac{F e_{2} O_{3}}{2 * F e}=\frac{159.7}{111.7}=1.43
$$

Inserting the relation of eqn (2) into eqn (1), results in:

$$
\frac{V_{o x}}{V_{m e t}}=2.13
$$

Repetition of this calculation for the other oxides gives a volumetric ratio of 2.10 for $\mathrm{Fe}_{3} \mathrm{O}_{4}$, and 2.02 for $\mathrm{Cr}_{2} \mathrm{O}_{3}$.

After removal of the passive layer, the bare metal surface is at the same height as the oxide-metal interphase of the remaining layer. Re-passivation implies that the metal surface has to be transformed to oxide. The remaining step of $-9 \mathrm{~nm}$ after passivation represents therefore the equivalent metal layer of the removed passive layer. Considering eqn (3), the volume of the oxidized metal is about 2.1 times the volume the metal ions would have in the metal lattice. Hence the thickness of the passive layer can be estimated at about $2.1 *-9$ $\mathrm{nm}=-18.9 \mathrm{~nm}$. This is close to the extrapolated initial step height of $-21 \mathrm{~nm}$. It must be realized that the metal-oxide interphase of the re-passivated area is now also $9 \mathrm{~nm}$ lower than the interphase of the not-sputtered area.

The density ratios calculated with eqn (1) are for crystalline materials. However, parts of the passive layer can be amorphous. The density of a fully amorphous oxide is about $10 \%$ lower of the crystalline value. The volumetric factor of eqn (3) will change to 2.39, resulting in an estimate of -21.5 nanometer of the passive film.

In future experiments, local height variations (aside from the sputter step) can be detected. They can originate from different sputter yields depending on crystal orientation, as shown for gold $\langle 110\rangle,<100\rangle$ and $<111\rangle$ directions [7]. Conversely, also passivation speeds and film thickness may depend on grain orientation.

\section{CONCLUSIONS}

In this work, we have demonstrated that:

- Recovery of the passive layer can be measured in-situ using AFM;

- The thickness of the passive film formed in air can be estimated to 21 nanometers;

- Recovery of this layer after sputtering takes about 2 hours.

\section{ACKNOWLEDGEMENTS}

This research was carried out under the project number T63.3.12480 in the framework of the research program of the Materials innovation institute M2i, Delft, the Netherlands.

The authors acknowledge prof. P. Rudolf of the Surfaces and Thin Films group, University of Groningen, for use of the XPS and argon-sputtering experimental setup. 


\section{REFERENCES}

[1] Jin, S. \& Atrens, A., Passive films on stainless steels in aqueous media. Appl. Phys. A., 50, pp. 287-300, 1990. doi:10.1007/BF00324495.

[2] Olsson, C.O.A. \& Landolt, D., Passive films on stainless steels - Chemistry, structure and growth, Electrochim. Acta, 48, pp. 1093-1104, 2003. doi:10.1016/S0013-4686 (02)00841-1.

[3] Ramachandran, D. et al., TEM and AES investigations of the natural surface nanooxide layer of an AISI 316L stainless steel microfibre. J. Microsc., 264, pp. 207-214, 2016. doi:10.1111/jmi.12434.

[4] Olefjord, I., Esca-studies of the composition profile of low temperature oxide formed on chromium steels-I. Oxydation in dry oxygen. Corros. Sci., 15, pp. 687-696, 1975. doi:10.1016/0010-938X(75)90033-5.

[5] Baer, D.R. et al., Comparison of the sputter rates of oxide films relative to the sputter rate of SiO2. J. Vac. Sci. Technol. A Vacuum, Surfaces, Film, 28, pp. 1060, 2010. doi:10.1116/1.3456123.

[6] Viefhaus, H., Hennesen, K., Lucas, M., Müller-Lorenz, E.M. \& Grabke, H.J., Ion Sputter Rates and Yields for Iron-, Chromium- and Aluminium Oxide Layers, Surf. Interface Anal., 21, pp. 665-672, 1994. doi:10.1002/sia.740210911.

[7] Franklin, R.E., Kirk, E.C.G., Cleaver, J.R.A. \& Ahmed, H., Channelling ion image contrast and sputtering in gold specimens observed in a high-resolution scanning ion microscope. J. Mater. Sci. Lett., 7, pp. 39-41, 1988. 